\title{
La fièvre de la vallée du Rift à Madagascar : risques d'infection pour le personnel d'abattoir à Antananarivo
}

\author{
H.G. Zeller ${ }^{1 *}$ H.T. Rakotoharinadrasana ${ }^{1}$ \\ M. Rakoto-Andrianarivelo ${ }^{1}$
}

\begin{abstract}
Mots-clés
Genre humain - Virus de la fièvre de la vallée du Rift - Bovin - Transmission des maladies - Contrôle de maladies Zoonose - Abattoir - Madagascar.
\end{abstract}

Résumé

\begin{abstract}
La première manifestation épizootique de la fièvre de la vallée du Rift (FVR) à M adagascar a été rapportée en 1990-1991. U ne enquête sérologique a été réalisée en janvier 1995 au niveau de l'abattoir principal d'Antananarivo pour déterminer les risques d'infection par le virus FVR chez le personnel. Des anticorps IgG FVR ont été rencontrés chez 12/126 employés (9,5 p. 100), en association dans 11 cas avec des anticorps IgM FVR. Tous les sujets positifs travaillaient dans la première partie de la chaîne d'abattage. Les 11 employés porteurs d'IgM représentaient 48 p. 100 du personnel du hall d'abattage. Ils ont été infectés très probablement par aérosol lors de l'abattage d'un animal virémique et n'ont manifesté aucune symptomatologie clinique. La surveillance hebdomadaire de 40 bovins à l'abattoir d'octobre 1994 à janvier 1995 a montré un taux de portage d'IgG FVR de 7,0 p. 100. Un seul animal a présenté des IgM FVR début décembre 1994. Des mesures préventives telles que le port obligatoire d'un masque au niveau de la chaîne d'abattage et, si possible, la vaccination du personnel à haut risque d'exposition sont recommandées.
\end{abstract}

La fièvre de la vallée du Rift (FVR) est une anthropozoonose d'origine virale à transmission vectorielle culicidienne atteignant principalement les bovins et les petits ruminants. Limitée au continent africain, elle provoque chez les animaux infectés une atteinte hépatique, des avortements et une mortalité des jeunes. L'homme peut être infecté lors de l'abattage d'animaux infectieux (par contact ou par inhalation) ou secondairement par piqûre de moustiques vecteurs. De ce fait, les éleveurs, les agents vétérinaires et les employés des abattoirs constituent des populations à risque. Si les formes asymptomatiques sont de loin les plus fréquentes chez l'homme, cette maladie est classiquement associée à un syndrome fébrile bénin, ainsi qu'à des céphalées, des myalgies, des arthralgies, des douleurs rétro-orbitaires, et à une asthénie importante. Des encéphalites, des formes hémorragiques mortelles ont été décrites ainsi que des complications oculaires $(8,15)$. Les flambées

Unité de Virologie, Institut Pasteur de Madagascar, BP 1274 Antananarivo 101, Madagascar

Tél. : 261202240165 ; fax : 261202241534

Email : zeller@pasteur.mg

* Auteur pour la correspondance épizoo-épidémiques survenues en Egypte en 1977-78 (7) et en Mauritanie en 1987 (5) et les résurgences en 1993 (2) ont révélé que la FVR était une anthropozoonose majeure.

A Madagascar, le virus de la FVR a été isolé pour la première fois à partir de moustiques capturés en décembre 1979 dans la région d'Andasibe $\left(48^{\circ} 25^{\prime}\right.$ S, $18^{\circ} 55^{\prime}$ E). L'isolement du virus s'est accompagné de la contamination de quatre personnes du laboratoire qui ont présenté des signes pseudo-grippaux (4). Des enquêtes sérologiques dans différentes régions de l'île avaient montré un faible niveau de circulation du virus de la FVR aussi bien chez l'homme que chez les animaux (6). La première épizootie associée à des cas humains a été rapportée dans le district de Fenoarivo-Est sur la côte Est en mars 1990 (12). En janvier 1991, l'épizootie a gagné la région des hauts-plateaux autour d'Antananarivo puis d'Antsirabe (11). Le virus FVR a été isolé à partir de produits d'avortements dans différentes fermes d'élevage. Depuis 1991, des surveillances de la FVR ont été menées dans les foyers épizootiques précités et à proximité des villages où avait été enregistrée une pathologie abortive, dans certaines zones à forte densité animale, et à l'abattoir frigorifique d'Antananarivo (prélèvements au hasard des bovins) (10). Aucune flambée épizootique n'a été décelée. 
La présente étude a porté sur l'évaluation des facteurs de risque d'exposition des employés à l'abattoir frigorifique d'Antananarivo par enquête sérologique. Les activités de l'abattoir, construit en 1972, ont démarré en juillet 1974. Cet abattoir a été entièrement réhabilité en 1990 et a été doté d'un agrément d'exportation des viandes pour les pays de la Communauté économique européenne.

\section{- SUJETS ET METHODES}

L'enquête s'est déroulée lors de la visite annuelle à la Médecine du travail, les 19 et 20 janvier 1995. Après consentement individuel, 126 parmi les 130 membres du personnel de l'établissement ont répondu à un questionnaire comportant notamment la date d'embauche à l'abattoir, les postes de travail successifs, les antécédents de vaccination, d'injection, de piqûres (par tiques, etc.), le contact avec diverses espèces animales en dehors du lieu de travail, les antécédents de maladies et d'hospitalisations éventuelles, les voyages en dehors de la région, et enfin les mesures de protection prises pendant le travail. Un prélèvement de sang veineux sur tube sec a été effectué. Un second prélèvement à trois semaines d'intervalle a été réalisé chez les employés porteurs d'anticorps FVR.

Les sérums, après centrifugation, ont été conservés à $4{ }^{\circ} \mathrm{C}$ jusqu'à traitement. Ils ont été testés par la technique ELISA d'immunocapture pour la détection d'anticorps $\operatorname{IgG/IgM~FVR~}(13,18)$. Pour la détection des IgG, la plaque Immulon II ${ }^{\mathrm{TM}}$ (Nunc, Danemark) a été sensibilisée avec un anticorps FVR de souris, l'antigène correspondant (souche AnMg 991) a été ajouté, puis les sérums à tester, à la dilution de screening $1: 100$, puis un conjugué anti $\operatorname{IgG} \gamma$ humaine couplé à la peroxydase (Kirkergaard \& Perry, Gaithesburg, USA). La fixation du conjugué a été révélée par addition de substrat chromogène ortho-toluidine et quantifiée au spectrophotomètre Titertek Multiscan IIтм. Un anticorps anti chaîne $\mu$ humain (Kirkergaard \& Perry) a été utilisé pour la sensibilisation des plaques pour la détection des $\operatorname{IgM}$, suivi de l'addition des sérums à tester au 1:100, puis de l'antigène FVR, d'un anticorps FVR de souris et enfin d'un conjugué anti IgG de souris couplé à la peroxydase (Kirkergaard \& Perry). Les titrages d'anticorps par dilution de série 2 des sérums ont été également réalisés. Pour les facteurs de risque, les comparaisons en proportion ont été effectuées avec le test exact de Fisher.

La confirmation des résultats obtenus par ELISA a été réalisée par séroneutralisation sur cellules Vero E6 comme précédemment décrit (16). Les sérums ont été testés aux dilutions $1 / 10$ à 1/1 280. Le titre correspond à la dilution neutralisant 100 doses infectantes de virus FVR (souche AnMg 991).

En parallèle, un prélèvement de sang a été effectué régulièrement chaque semaine d'octobre 1994 à janvier 1995 sur 40 bovins arrivant à l'abattoir pour recherche d'anticorps FVR.

\section{RESU LTATS}

La distribution des tâches des employés a permis de regrouper les différents départements en trois catégories selon le degré d'exposition à la FVR (tableau I) :

- groupe A (exposition maximale) : personnel en contact permanent avec des animaux vivants et aussi des viandes fraîchement abattues (section parc, hall d'abattage, triperie et boyauderie, frigorifique et couloir, sous-produits) ;
- groupe B (exposition modérée) : personnel en contact avec des carcasses abattues $24 \mathrm{~h}$ auparavant ou bien des carcasses réfrigérées (section découpe et manutention);

- groupe C (pas d'exposition) : absence de contact direct avec la carcasse et/ou les zébus vivants (section administration, cantine, menuiserie, sécurité, lingerie et transport).

Les employés de la section A étaient en moyenne plus âgés que les employés des sections $\mathrm{B}$ et $\mathrm{C}$ et travaillaient à l'abattoir depuis un plus grand nombre d'années.

Des anticorps IgG FVR ont été détectés chez 12 sur les 126 employés testés $(9,5$ p. 100) (tableau I) et confirmés par séroneutralisation (tableau II). Des IgM FVR ont été observées dans 11 cas (8,7 p. 100), en association avec des IgG FVR (tableau II). Tous les employés positifs travaillaient dans la section A de l'abattoir, dont 11 dans le hall d'abattage sur 23 personnes, soit 48 p. 100 de l'effectif, et 1 au niveau du parc. Parmi les 9 employés chez lesquels un second sérum a été obtenu, le titre d'IgM était stable pour 8 d'entre eux et en diminution dans 1 cas. Aucun contact des sujets positifs avec des animaux en dehors du milieu de travail n'a été rapporté lors de l'enquête, confirmant la contamination à l'abattoir.

\section{Tableau I}

Répartition des employés de l'abattoir d'Antananarivo par groupes d'exposition et postes de travail, et résultats des sérologies IgG/IgM FVR par ELISA réalisées en janvier 1995

\begin{tabular}{|c|c|c|c|c|}
\hline $\begin{array}{l}\text { G roupes } \\
\text { d'exposition }\end{array}$ & Sections & $\begin{array}{c}\text { Employés } \\
\text { Nb. }\end{array}$ & $\begin{array}{l}\text { FVR IgG } \\
\text { Nb. (\%) }\end{array}$ & $\begin{array}{l}\text { FVR IgM } \\
\text { Nb. (\%) }\end{array}$ \\
\hline \multirow[t]{6}{*}{ A } & Parc & 8 & $1(0,3)$ & \\
\hline & Tuerie & 4 & & \\
\hline & $\begin{array}{l}\text { Hall } \\
\text { d'abattage }\end{array}$ & 23 & $11(47,8)$ & $11(47,8)$ \\
\hline & $\begin{array}{l}\text { Frigorifique } \\
\text { et couloir }\end{array}$ & 4 & & \\
\hline & $\begin{array}{l}\text { Triperie et } \\
\text { boyauderie }\end{array}$ & 10 & & \\
\hline & Sous-produits & 2 & & \\
\hline Sous-total A & & 51 & $12(23,5)$ & $11(21,5)$ \\
\hline \multirow[t]{3}{*}{ B } & $\begin{array}{l}\text { Découpe et } \\
\text { manutention }\end{array}$ & on & & \\
\hline & $\begin{array}{l}\text { Parage et } \\
\text { désossage }\end{array}$ & 28 & & \\
\hline & $\begin{array}{l}\text { Servant } \\
\text { réfrigération }\end{array}$ & n & & \\
\hline Sous-total B & & 39 & & \\
\hline \multirow[t]{4}{*}{ C } & M aintenance & e 11 & & \\
\hline & $\begin{array}{l}\text { Cantine et } \\
\text { lingerie }\end{array}$ & 6 & & \\
\hline & Sécurité & 13 & & \\
\hline & $\begin{array}{l}\text { Adminis- } \\
\text { tration }\end{array}$ & 6 & & \\
\hline Sous-total C & & 36 & & \\
\hline Total & & 126 & $11(8,7)$ & $12(9,5)$ \\
\hline
\end{tabular}




\section{Tableau II}

Titrage des anticorps FVR par ELISA et séroneutralisation chez les employés positifs de la section A, sur deux sérums prélevés à trois semaines d’intervalle (janvier 1995)

\begin{tabular}{|c|c|c|c|c|c|c|c|}
\hline \multirow{3}{*}{ Postes de travail } & \multirow[b]{3}{*}{ Titres : } & \multicolumn{3}{|c|}{ Sérum 1} & \multicolumn{3}{|c|}{ Sérum 2} \\
\hline & & \multicolumn{2}{|c|}{ ELISA } & \multirow[t]{2}{*}{ SN } & \multicolumn{2}{|c|}{ ELISA } & \multirow[t]{2}{*}{ SN } \\
\hline & & $\lg M$ & IgG & & $\operatorname{Ig} M$ & IgG & \\
\hline Dépouillage & & 3200 & 800 & 160 & 3200 & 800 & 40 \\
\hline Parage & & 3200 & 1600 & nt & 1600 & 800 & $\mathrm{nt}$ \\
\hline Eviscération & & 1600 & 1600 & 20 & 1600 & 1600 & 40 \\
\hline Dépouillage & & 400 & 800 & 160 & nt & $\mathrm{nt}$ & nt \\
\hline Dépouillage & & 1600 & 3200 & 80 & 1600 & 3200 & 80 \\
\hline Dépouillage & & 400 & 3200 & 80 & 800 & 6400 & 80 \\
\hline Eviscération & & 1600 & 800 & $\mathrm{nt}$ & nt & nt & $\mathrm{nt}$ \\
\hline Dépouillage & & 3200 & 3200 & nt & 3200 & 3200 & 160 \\
\hline Découpe tête et abats & & 400 & 800 & 80 & 400 & 80 & 40 \\
\hline Découpe des carcasses & & 400 & 6400 & nt & 400 & 12800 & 160 \\
\hline Découpe des carcasses & & 400 & 3200 & 160 & nt & nt & $\mathrm{nt}$ \\
\hline
\end{tabular}

SN : séroneutralisation ; nt : non testé

Aucun antécédent clinique évocateur d'atteinte par le virus FVR n'a été rapporté au niveau de l'ensemble du personnel. Il n'y pas eu de manifestations cliniques rapportées pour les 11 employés qui ont été infectés récemment. La prévalence de sujets porteurs d'IgG FVR est significativement plus forte dans le groupe A $\left(\mathrm{p}<10^{-5}\right)$. Il en va de même pour les sujets porteurs d'IgM FVR $\left(\mathrm{p}<10^{-4}\right)$.

Au niveau du cheptel, sur les 600 animaux testés d'octobre 1994 à janvier 1995, 42 (7 p. 100) avaient des anticorps IgG FVR dont un seul en association avec des IgM spécifiques, début décembre 1994.

\section{DISCUSSION}

L'enquête a été réalisée dans le seul abattoir d'Antananarivo construit selon les normes de bonne pratique de manipulation, avec utilisation de matériels adéquats, une distribution des tâches stable et bien structurée, des mesures d'hygiène appropriées et des contrôles réguliers. La technique utilisée pour la détection des $\operatorname{IgG}$ et IgM FVR est spécifique de la FVR, d'autant qu'à Madagascar aucun autre Phlebovirus n'a été isolé jusqu'à présent et aucune vaccination FVR vaccin vivant atténué ou inactivé n'a été pratiquée. Chez l'homme, les IgM FVR qui apparaissent dans la semaine suivant l'infection atteignent leur maximum aux alentours $\mathrm{du} 10^{\mathrm{e}}$ jour, restant en plateau pendant un à deux mois environ avant de disparaître définitivement. Les anticorps $\operatorname{IgG}$ qui apparaissent peu de temps après les IgM persistent plusieurs années.

La présence d'anticorps IgM FVR chez 11/126 employés de l'abattoir témoigne d'une atteinte récente par le virus de la FVR. Les employés séropositifs appartenaient tous au premier groupe d'exposition et, plus précisément, les employés porteurs d'IgM FVR provenaient uniquement de la section du hall d'abattage et étaient exposés au sang frais. Il semble probable que le personnel ait été infecté en décembre, période correspondant au début de la saison des pluies, période de transmission propice du virus comme l'ont montré les isolements à partir de moustiques en décembre 1979 et les manifestations épizootiques de 1990-1991 (4, 10). L'origine la plus probable de la contamination serait un ou plusieurs animaux virémiques asymptomatiques (d'un même lot) à l'abattage. La virémie chez l'animal dure environ quatre jours (17) et la concentration virale peut être très élevée jusqu'à $10^{10}$ parti- cules par ml de sang (8). L'absence de manifestations cliniques au niveau du personnel a rendu l'infection inapparente. La contamination des employés du hall d'abattage a pu se faire de deux façons, soit par inhalation de particules infectées sous forme d'aérosol à partir du sang, soit par contact direct entre sang infecté et micro-lésions ou blessures de la peau. Si l'utilisation de gants voire de mono-gant métallique protège les mains de blessures par le couteau et limite la contamination par contact, l'absence de masque de protection avait été constatée lors de l'enquête. La source de contamination ayant été ponctuelle, la liaison avec l'âge n'est que le reflet de la liaison entre l'âge et l'appartenance à l'un des lieux de travail.

Dans une enquête réalisée dans un abattoir de bétail à Lusaka en Zambie, des anticorps avaient été observés chez 5 sur 53 personnes (9,4 p. 100). Par contre, parmi les 40 personnes d'un abattoir de porcs, aucune n'avait d'anticorps FVR (8). Une autre enquête sérologique similaire réalisée en 1979 dans deux abattoirs à Sinoia et Gatooma au Zimbabwe (3) par technique d'inhibition d'hémagglutination avait montré que sur 365 employés 18 p. 100 de ceux appartenant au groupe à exposition élevée étaient positifs pour IgG FVR, contre 3,2 et 2,9 p. 100 dans les groupes à exposition modérée ou nulle. En Egypte, une étude similaire durant l'épizootie de 1993 a montré une prévalence significativement plus élevée chez les employés travaillant à des postes à haut risque (1). Aucun épisode fébrile chez les personnes positives n'a été relevé dans les deux mois précédant l'enquête. Il a été suggéré que les employés d'abattoir pourraient constituer une population sentinelle pour la surveillance de la fièvre de la vallée du Rift (1).

Les résultats de toutes ces enquêtes montrent que le groupe d'employés en début de chaîne d'abattage, en contact direct avec le sang et les carcasses fraîches, présente un risque important de contracter la FVR. Le risque d'exposition reste cependant limité. A Antananarivo, un seul employé avait été antérieurement infecté, soit 1,9 p. 100 des personnes du groupe exposé qui travaillaient pour la plupart depuis plus de 10 ans au sein de cette section.

La grande majorité des animaux provenaient du marché au bétail le plus important du pays, Tsiranomandidy, qui drainait une grande partie du territoire. Il est donc impossible de préciser l'origine géographique de la contamination des animaux, sachant que la plupart d'entre eux cheminent à pied depuis leur zone d'élevage (14). En 
décembre 1994, un bovin était porteur d'IgM FVR, signe de circulation du virus à bas bruit et un ou plusieurs animaux virémiques ont infecté 47 p. 100 des employés dans le hall d'abattage, principalement par aérosol. La recommandation du port de masque a été faite pour minimiser les risques d'infection. La vaccination du personnel à risque serait une mesure préventive appropriée mais impossible à réaliser étant donné l'absence de vaccin à usage humain disponible.

\section{Remerciements}

Nous tenons à remercier la direction des Abattoirs frigorifiques d'Antananarivo qui nous a permis d'effectuer cette enquête.

\section{BIBLIO GRAPH IE}

1. ABU -ELYAZEED R., EL-SHARKAWY S., OLSON J., BOTROS B., SO LIMAN A., SALIB A., CUMM IN GS C., ARTHUR R., 1996. Prevalence of anti-Rift Valley fever IgM antibody in abattoir workers in the Nile Delta during the 1993 outbreak in Egypt. Bull. WHO, 74: 155-158.

2. ARTHUR R.R., EL-SHARKAWY M.S., COPE S.E., BOTROS B.A OUN S., MORRILL J.C., SHOPE R.E., HIBBS R.G., DARWISH M.A., IMAM I.Z., 1993. Recurrence of Rift Valley fever in Egypt. Lancet, 342: 1149-1150.

3. CHAM BERS P.G., SWANEPOEL R., 1980. Rift Valley fever in abattoir workers. Cent. Afr. J. Med., 26: 122-126.

4. CLERC Y., RASO LOFONIRINA N., DIGOUTTE J.P., RODHAIN F. COULAN GES P., 1981. Infection humaine à virus Zinga et $Y 251$ (soustype de Sindbis). Arch. Inst. Pasteur Madagascar, 50 : 60-67.

5. JOUAN A., LEGUENNO B., DIGOUTTE J.P., PHILIPPE B., RIUO 0. ADAM F., 1988. An RVF epidemic in Southern Mauritania. Ann. Virol. 139: 307-308.

6. MATHIOT C., FONTENILLE D., GEORGES A.J., COULANGES P., 1989. Antibodies to haemorrhagic fever viruses in Madagascar. Trans. R. Soc. trop. Med. Hyg., 83: 407-409.

\section{Summary}

Zeller H.G., Rakotoharinadrasana H.T., Rakoto-Andrianarivelo M Rift Valley fever in Madagascar: infection risks for the abattoir staff in Antananarivo

The first Rift Valley fever (RVF) epizootic was reported in Madagascar in 1990-1991. A serological survey was conducted in Antananarivo main slaughterhouse to determine the risks of infection by the RVF virus among workers in January 1995. In 12 out of 126 workers (9.5\%), RVF IgG antibodies were detected in association with RVF IgM antibodies in 11 cases. All positive individuals worked in the first section of the slaughter line. The 11 RVF IgM positive workers represented $48 \%$ of the staff in that area. It is most likely that they were infected by an aerosol during the slaughter of a viremic animal. They did not show any clinical symptoms. Another serological survey conducted in 40 bovines every week in the abattoir from 0 ctober 1994 to January 1995 showed a 7.0\% RVF IgG prevalence rate. O nly one animal was reported RVF IgM positive early December 1994. Preventive measures such as mandatory mask wearing on the slaughter line and if possible vaccination of high risk staff are recommended.

Key words: M ankind - Rift Valley fever virus - Cattle - D isease transmission - Disease control - Zoonosis - Abattoir Madagascar.
7. MEEGAN J.M., 1979. The Rift Valley fever epizootic in Egypt 1977-78. 1. Description of the epizootic and virological studies. Trans. R. Soc. trop. Med. Hyg., 73: 618-623.

8. MEEGAN J.M., BAILEY C.H., 1988. Rift Valley fever. In: Monath T. ed. The arboviruses, epidemiology and ecology, Vol. 4. Boca Raton, $\mathrm{FI}$ USA, CRC Press, p. 51-76.

9. MORITA C., 1988. Prevalence of Rift Valley fever in Lusaka and Mazabuka. Zambia J. vet. Med., 35: 157-160.

10. MORVAN I., ROLLIN P.E., LAVENTURE S., RAKOTOARIVONY I., ROUX J., 1992. Rift Valley fever epizootic in the central highlands of Madagascar. Res. Virol., 143: 407-415.

11. MORVAN J., ROLLIN P.E., ROUX J., 1992. Situation de la fièvre de la vallée du Rift à Madagascar en 1991. Enquêtes séro-épidémiologiques chez les bovins. Revue Elev. Méd. vét. Pays trop., 45 : 121-127.

12. MORVAN J., SALUZZO J.F., FONTENILLE D., ROLLIN P.E. COULANGES P., 1991. Rift Valley fever in the East Coast of Madagascar. Res. Virol., 142: 475-482.

13. NIKALSSON B., PETERS C.J., GRANDIEN M., WOOD O., 1984 Detection of human immunoglobulins $G$ and $M$ antibodies to Rift Valley fever virus by enzyme-linked immunosorbent assay. J. clin. Microbiol., 19: 225-229.

14. RIBOT J.J., ANDRIAM AHEN INA E.F., 1974. La commercialisation du bétail de Tsiroanomandidy à Tananarive. Bull. Acad. malg., 51 : 237-238.

15. SIAM A.L., MEEGAN J.M., 1980. O cular disease resulting from infection with Rift Valley fever virus. Trans. R. Soc. trop. Med. Hyg., 74: 539-541.

16. SWANEPOEL R., STRUTHERS J.K., ERASMUS M.J., SHEPERD S.P., MCGILLIVRAY G.M., 1986. Comparison of techniques for demonstrating antibodies to Rift Valley fever. J. Hyg. Camb., 97: 317-329

17. TURELL M.J., BAILEY C.L., ROSSI C.A., 1984. Increased mosquito feeding on Rift Valley fever virus infected lambs. Am. J. trop. Med. Hyg., 33: $1232-1238$.

18. ZELLER H.G., BESSIN R., THIONGANE Y., BAPETEL I., TEOU K., GBAGUIDI ALA M., NDE ATSE A., SYLLA R., DIGOUTTE J.P., AKAKPO J.A., 1995. Rift Valley fever antibody prevalence in domestic ungulates in Cameroon and several West African countries (1989-1992) following the 1987 Mauritanian outbreak. Res. Virol., 146: 81-85.

Reçu le 24.4.97, accepté le 17.3.98

\section{Resumen}

Zeller H.G., Rakotoharinadrasana H.T., Rakoto-Andrianarivelo M. La fiebre del valle del Rift en Madagascar: riesgos de infección para el personal del matadero de Antananarivo

Entre 1990-1991, se reportó la primera manifestación epizoótica de la fiebre del valle del Rift (FVR) en Madagascar. En enero de 1995 se llevó a cabo una encuesta serológica, a nivel del matadero principal de Antananarivo, con el fin de determinar los riesgos de infección por el virus FVR en el personal. Anticuerpos lgG FVR se encontraron en $12 / 126$ de los empleados $(9,5 \%)$, en 11 de los casos asociación con anticuerpos IgM FVR. Todos los individuos positivos trabajaban en la primera etapa de la cadena de sacrificio. Los 11 empleados portadores de IgM representaron $48 \%$ del personal del corredor de matanza. La infección se obtuvo probablemente por aerosoles, durante la matanza de un animal virémico y no hubo manifestaciones clínicas de sintomatología. La observación semanal de 40 bovinos en el matadero, de octubre 1994 a enero 1995, mostró una tasa de positividad para IgG FVR de 7.0\%. Unicamente un animal presentó IgM FVR a inicio de diciembre 1994. Se recomiendan medidas preventivas, como el uso obligatorio de una máscara en la cadena de matanza y, de ser posible, la vacunación del personal a alto riesgo de exposición.

Palabras clave: Genero humano - Virus fiebre del Valle del Rift - Ganado bovino - Transmisión de enfermedades - Control de enfermedades - Zoonosis - M atadero - M adagascar. 